\title{
Mechanical properties of tungsten wires after cycling deformation treatment
}

\author{
Wlodzimierz Bochniak • Krzysztof Piela • \\ Stanislau Vinarski • Marek Lagoda
}

Received: 9 April 2013 / Accepted: 28 June 2013 /Published online: 14 July 2013

(C) The Author(s) 2013. This article is published with open access at Springerlink.com

\begin{abstract}
This article presents the influence of cycling deformation treatment executed by continuous multilateral bending with shearing on the mechanical and technological important properties of tungsten wire intended for production of spiral shapes. The mechanical properties are determined by a simple uniaxial tensile test while the technological capability is based on torsion with tension test (TT test). It is shown that the cycling deformation treatment of the wire practically does not change its mechanical parameters, but significantly increases its technological properties. The results therefore indicate an inadequacy of a simple tension test for selection (quality assessment) of tungsten wires.
\end{abstract}

Keywords Tungsten $\cdot$ Wire $\cdot$ Properties $\cdot$ Bending . Manufacturing $\cdot$ Parameters $\cdot$ Processes

\section{Introduction}

Tungsten wires, with a thickness from several to tens of micrometers, are plastically formed into spirals and used for incandescent and discharge light sources. Wire manufacturing is based on the powder technology, i.e., tungsten powder obtained through a chemical process is subjected successively to pressing, sintering, and plastic forming (rotary forging and drawing). Note that the wire-winding process needs to result in good plastic properties and "not too high" elasticity. On the other hand, due to the exploitation conditions of spirals, and above all, the required high-creep resistance, recrystallized wires are not suitable for production, especially if they have a coarsegrained structure.

W. Bochniak $\cdot$ K. Piela $\cdot$ S. Vinarski $\cdot$ M. Lagoda $(\bowtie)$ Department of Structure and Mechanics of Solids, Faculty of NonFerrous Metals, AGH-University of Science and Technology, A. Mickiewicza Av. 30, 30-059 Cracow, Poland

e-mail:mlagoda@agh.edu.pl
Modifying the mechanical and plastic properties of metallic materials, in particular, reducing the strong work hardening without an annealing treatment is possible using mechanical training. This process consists of subjecting the metal to repeated, alternating, and low-plastic deformation. The effects of cyclic contraflexure on mechanical properties of metals are documented, among others, in Bochniak and Mosor's [1] paper, herein using CuSn $6.5 \%$ tin bronze strips. It was shown that mechanical training leads to a work softening.

Unfortunately, the mechanical parameters of tungsten wires determined in simple uniaxial tensile tests are far insufficient to predict their behavior in the production process of spirals. These wires, in spite of similar mechanical properties, are often characterized by significantly different susceptibility to winding. Therefore, when assessing the technological characteristics of tungsten wire, the results of the following tests are considered to be more reliable: core wire winding, unidirectional torsion, knife-edge compression, bend-and-stretch, or reversible banding [2]. Recently, a new technological test was proposed [3], in which the wire is subjected to simultaneous torsion with tension (TT test), and the stress state - in the opinion of the authors - is close to that which occurs in the production process of the filaments. Moreover, the results of TT tests conducted on tungsten wires with different diameters have shown its ability to anticipate their later behavior during technological processes $[4,5]$.

The aim of the work presented herein is to answer the question of whether, and if, to what extent the use of cycling deformation treatment (CDT) on tungsten wire by continuous multilateral bending with shearing method [6], may modify its mechanical and technological important properties.

Generally speaking, the cyclic deformation of metals (e.g., by tension and compression or bilateral bending) may be accompanied by two different structural processes. The first is characteristic for the deformation with small amplitudes and 


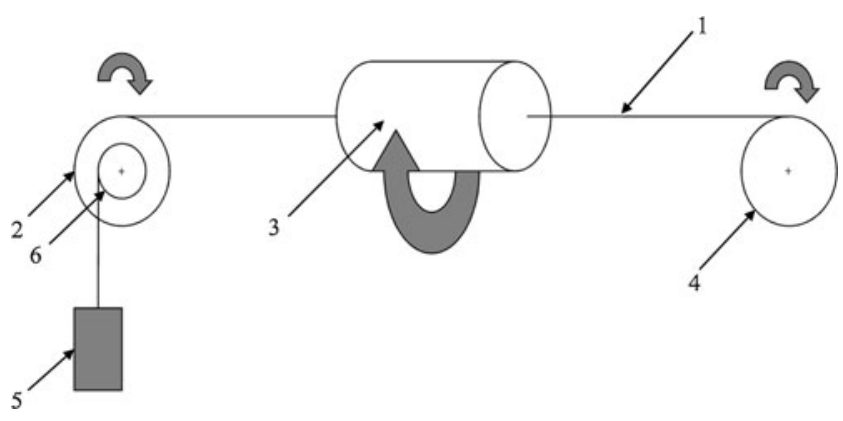

Fig. 1 Scheme of the process of mechanical training of wires: 1 tungsten wire, 2 coil with wire to unreel, 3 system of six rotating dies, 4 winding coil, 5 break weight, and 6 brake (steel cylinder with a band of tin bronze around it)

involves so-called fatigue phenomena, resulting in the strongly work-hardened metal turning into a strain-softened one before its destruction occurs [7].

The second process, dominant during deformation with high-strain amplitudes, produces strong heterogenization of plastic flow-generating shear bands. Consequently, there is a drastic fragmentation of the metal structure, in particular, the formation of nano-sized grains, thus, a significant increase in its mechanical properties at the expense of workability. Such an effect is obtained in e.g., continuous repetitive corrugation and straightening method developed by Huang et al. [8], which consists of multiple, alternate, passing (rolling) of strips between the "geared" and smooth rolls, or in a more sophisticated way, which is a method of continuous bending under tension [9], where the stretched strip is contraflexed due to a reversible movement along its length of set of rotating rolls. Of course, the extensive fragmentation of grains can also be obtained during monotonic deformation with large strain, using the so-called Severe Plastic Deformation methods, in particular, methods of Equal Channel Angular Extrusion [10] most often satisfying the conditions for simple shear of metal. Unfortunately, they are mainly used on the laboratory scale and it is technically not possible to use them to obtain specific mechanical properties of long strips or wires.

Some attempts have also been done to assess the influence of cyclically changing shear applied with small unit deformations on the ability to activate the fatigue phenomena. The results of experimental studies carried out [11] on strips of copper and cobalt by contraflexure with shearing confirmed the above thesis. Although the contraflexure with shearing method is fairly easy to be applied to flat metallic parts, the more direct application for wires does not make sense, because, by definition, it does not guarantee obtaining homogeneous structure, and thus identical properties on the circumference (with arbitrarily oriented radius) of the wire. For this reason, this paper utilizes a newly formed and original method of CDT designed for thin wires, based on continuous multilateral bending with shearing.

\section{Experiment}

CDT of tungsten wire with a diameter of $200 \mu \mathrm{m}$ was performed on a specially constructed test device whose scheme is shown in Fig. 1. Unreeled wire (1) from the coil (2) with diameter of $100 \mathrm{~mm}$, was introduced into a system of six dies (3), with holes of the same diameter as the wire, which are fixed in a common housing and rotating around the axis at a speed of $1,350 \mathrm{rev} / \mathrm{min}$. After passing through the device, the wire was reeled on the coil (4) with a diameter of $100 \mathrm{~mm}$ rotating at a speed of $115 \mathrm{rev} / \mathrm{min}$. Applied parameters decide of the linear speed of wire relative to the rotating dies is $26.8 \mathrm{~mm} / \mathrm{rev}$.

Appropriate design of the dies system meant that every second die revolved eccentrically (Fig. 2), and each piece of wire passing through the rotating dies was subjected to continuous multilateral bending with shearing inducted by ironing at the edge of inner surface of the dies.
Fig. 2 Schematic layout of the rotating dies (labeled with number 3 in Fig. 1)

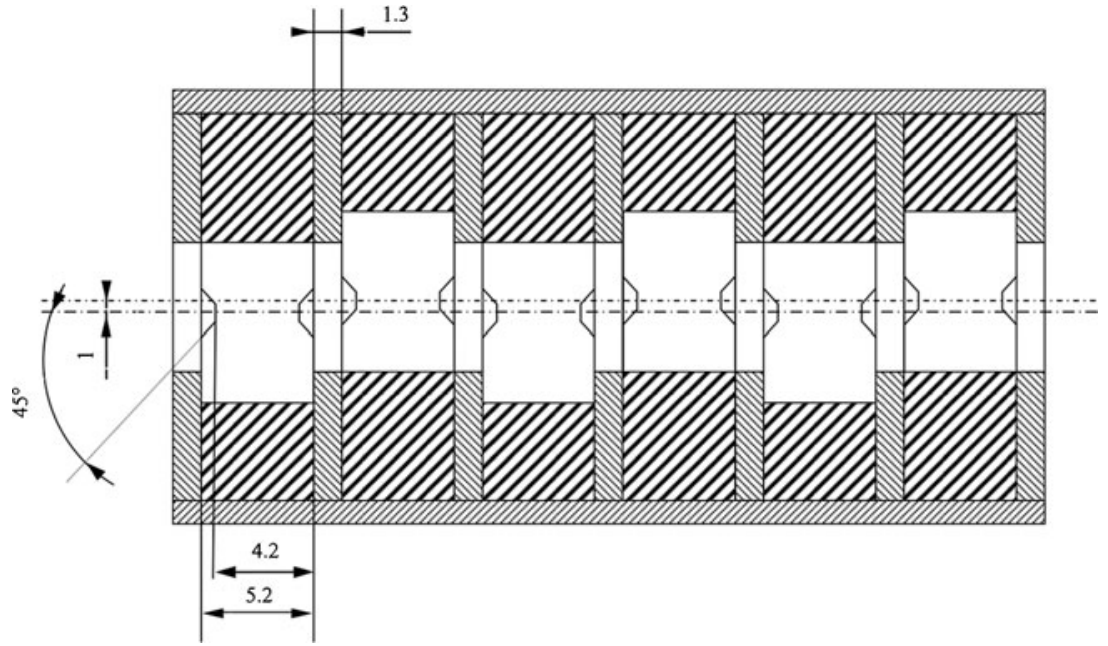


Fig. 3 System of dies: a general view; $\mathbf{b}$ basic parts: 1 centric dies, 2 eccentric dies, 3 spacer rings
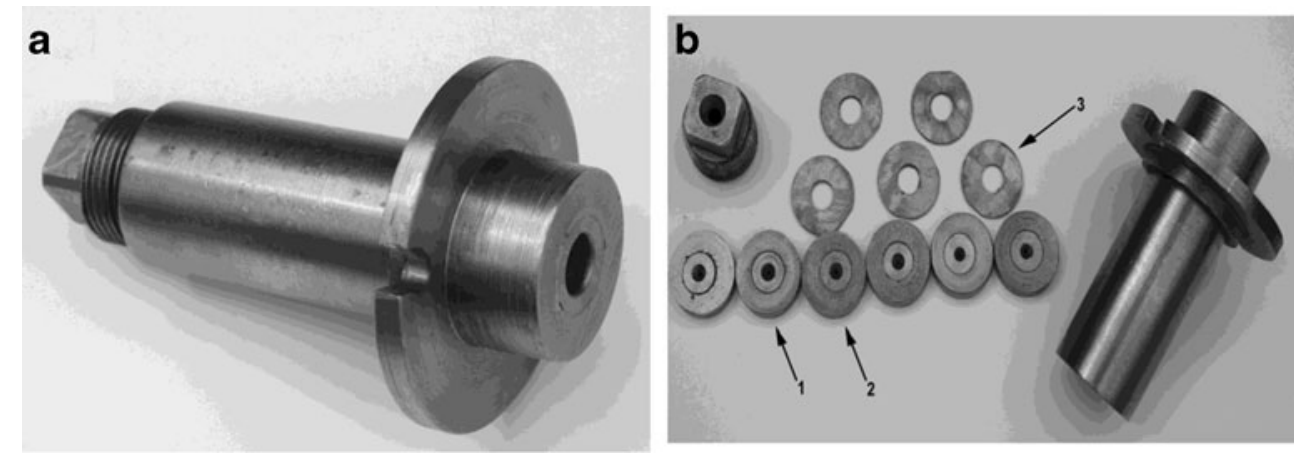

Unreeled wire was under the influence of initial stress due to application of tension, which not only protects it from entanglement, but also determines mutual participation of bending and shearing deformation. This was possible to achieve thanks to the brake mounted onto the coil in the form of a tin bronze strip pressed by a weight (designated as 5 and 6 in Fig. 1). Figure 3 shows the appearance of the device training when folded, and each of its components. Training of wires was performed with two different weights: 4.7 and $8.5 \mathrm{~N}$, up to four passes through the set of dies. Axial stress amounted respectively to the 150 and $270 \mathrm{MPa}$.

Tensile test of wire (both in initial state and trained) were performed on the Zwick Roell testing machine. Samples gauge length was $100 \mathrm{~mm}$ and tensile strain rate was $8 \times 10^{-3} \mathrm{~s}^{-1}$. In each case, one measurement point (for each of the variants) represents at least five samples.

TT test was performed on a special apparatus whose scheme is shown in Fig. 4 earlier presented by Bochniak et al. (2010). The center of the tungsten wire (1) with a length of $1 \mathrm{~m}$ was placed in a catch (2), and then its ends, after passing through the guide rolls (3), and attaching weights (4) of $10 \mathrm{~N}$ each, were blocked in a clamp (5). The rotary motion of the catch (2) resulted in the winding two pieces of wire

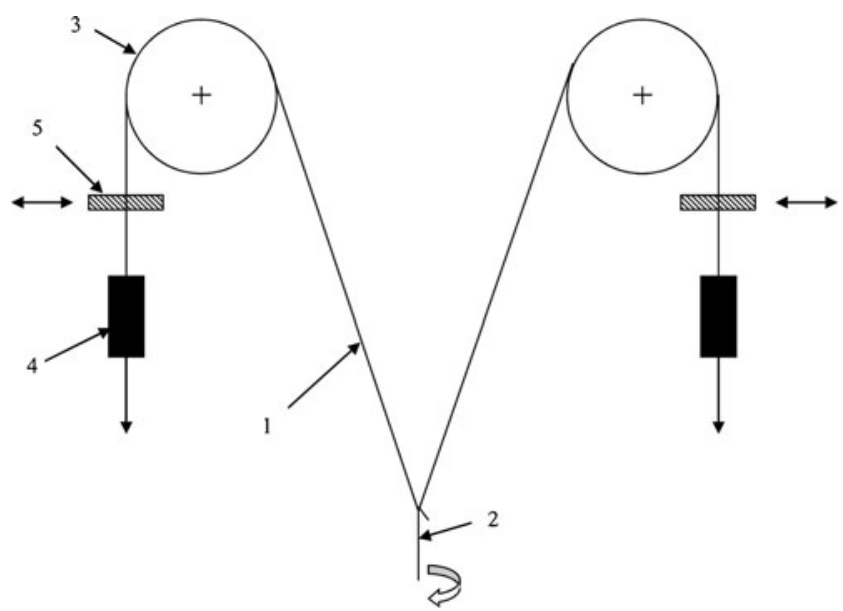

Fig. 4 Scheme of the TT test: 1 tested wire, 2 catch rotated by an electric motor, coupled with the twist recording device, 3 guide rolls, 4 weights, 5 jaws clamping the ends of wire (reeled on themselves), with fixed ends of the tested sample, was carried out with a gradual increase of tensile stresses.

The test result was the number of twists $\left(\mathrm{N}_{\mathrm{T}}\right)$ needed to rupture the wire and usually occurred on the front of the formed tangle, as shown in Fig. 5. At least ten tests per variant were performed. After the training, the wire had a slight wavy shape. It should be emphasized that according to papers of Bochniak and Pieła (2007) [4] and Filipek (2010) [5] the TT test is a simple, fast, and cheap method to determine the technological properties of wires intended for winding.

\section{Results}

The effect of initial tension and the number of passes in the CDT process on the properties of tungsten wires are shown in Figs. 6 and 7. A large scatter of obtained mechanical parameters of wire illustrate the scale of inhomogeneity of the material obtained by powder technology, and therefore, the analysis carried out focuses on the trends of changes of tested properties and not on their absolute values.

Commercial tungsten wire is characterized by average values of yield stress (YS) equal to 2,026 MPa, ultimate tensile strength (UTS) of $2,294 \mathrm{MPa}$, total elongation of $A \approx 2.6 \%$ and the $\mathrm{N}_{\mathrm{T}}$ as much as 28 . Regardless of the magnitude of the applied tension, CDT results in only a small decrease of UTS (not exceeding $3 \%$ for the wire after four passes), and both YS and $A$ remain relatively at the same level (Figs. $6 \mathrm{a}-\mathrm{c}$ and $7 \mathrm{a}-\mathrm{c}$ ).

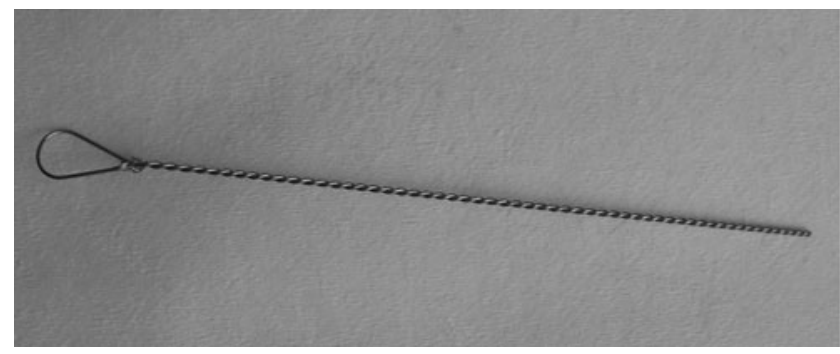

Fig. 5 View of the tungsten wire after fracture in the TT test 
a

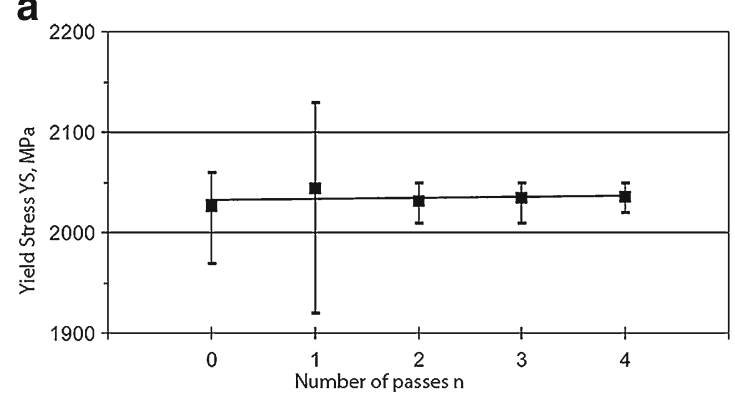

C

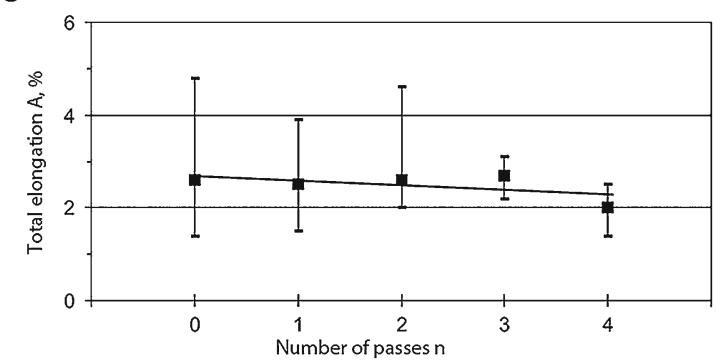

b

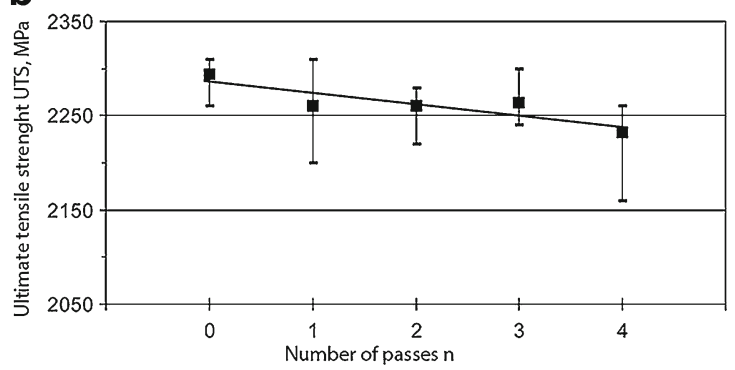

d

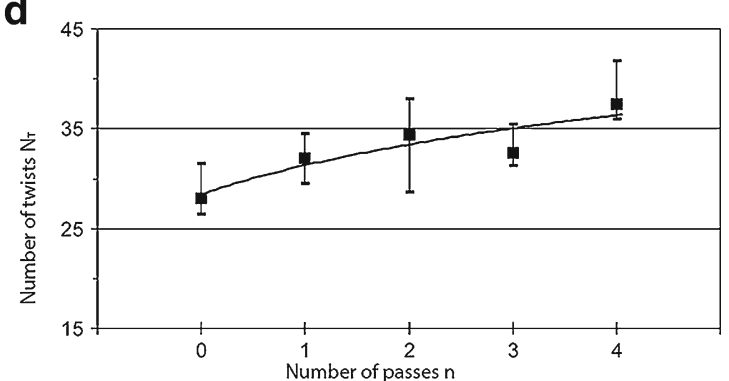

Fig. 6 Effect of mechanical training (number of passes $n$ ) on mechanical (a-c) and technological (d) (defined by $\mathrm{N}_{\mathrm{T}}$ in the TT test) properties of tungsten wire; attached weight value of $4.7 \mathrm{~N}$

CDT always leads to a significant increase in the number of wire twists $\mathrm{N}_{\mathrm{T}}$. In particular, for the first two passes, $\mathrm{N}_{\mathrm{T}}$ reaches more than 34 for a tension of $4.7 \mathrm{~N}$ and almost 33 for a tension of $8.5 \mathrm{~N}$. This represents an increase of approximately $20 \%$ with respect to the commercial wire. Applying a higher number of passes leads to a further increase in $\mathrm{N}_{\mathrm{T}}$ only in the case of training under tension of $4.7 \mathrm{~N}$. The wire after four passes shows the average magnitude of $\mathrm{N}_{\mathrm{T}}$ exceeding 37 , which, compared to the wire in initial state, represents an increase of over $30 \%$. Further training of the wire at higher

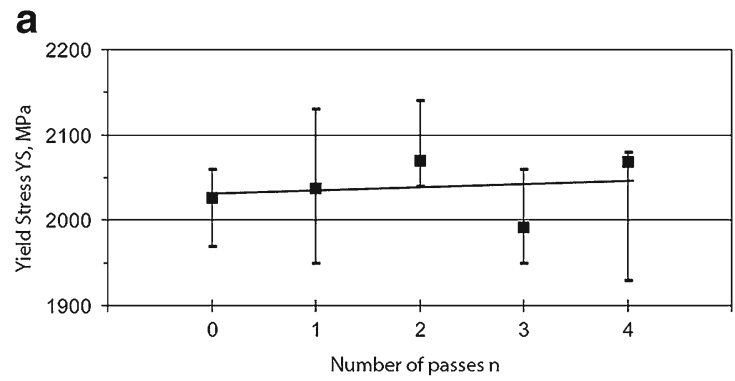

\section{C}

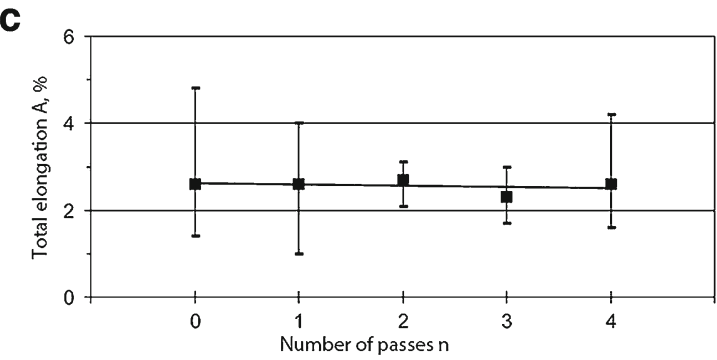

tensions would no longer change the magnitude of previously achieved $\mathrm{N}_{\mathrm{T}}$ values (Figs. $6 \mathrm{~d}$ and $7 \mathrm{~d}$ ).

\section{Analysis}

The obtained results show that the method used for tungsten wire CDT practically does not change its mechanical parameters determined in tensile tests (there was only a slight decrease in ultimate tensile strength), but significantly increased its
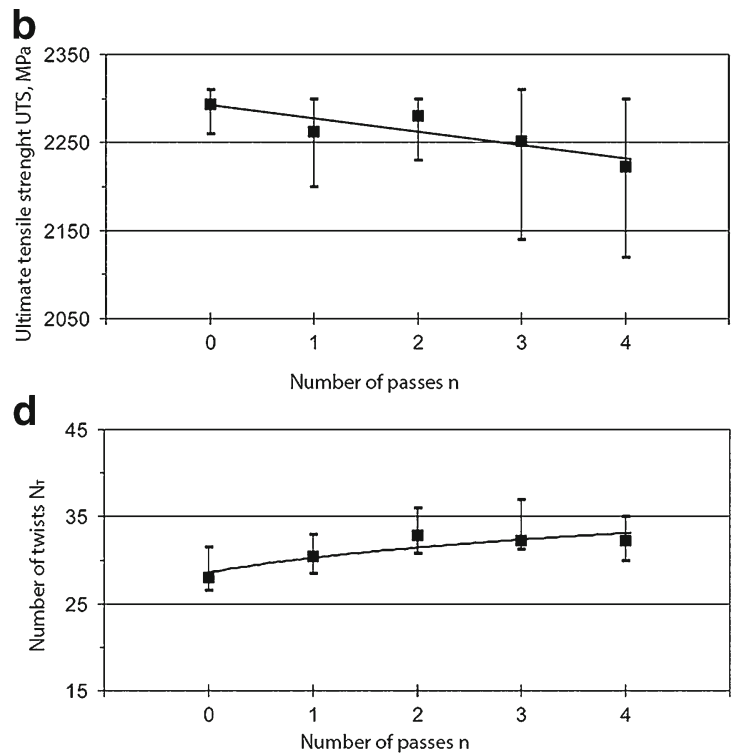

Fig. 7 Effect of mechanical training (number of passes $n$ ) on mechanical (a-c) and technological (d) (defined by $\mathrm{N}_{\mathrm{T}}$ in the TT test) properties of tungsten wire; attached weight value of $8.5 \mathrm{~N}$ 
technological properties intend for a spirals production; this is represented by the number of twists in the TT test. This confirms the results of earlier studies by Bochniak and Pieła (2007) [4] about the lack of convergence of the tensile test results with the observed behavior of wires in the production process of spirals.

The reaction of tungsten wires on the process of CDT significantly depends on the applied tension. At low-tension force, one observes a parabolic growth in the number of twists with the number of passes, while the application of larger values of tension leads (already after two passes) to achieving the state of saturation and the stabilization of previously obtained technological properties (Figs. 6d and 7d).

Such a diversified response of the tungsten wire underlines the fact that the magnitude of tension determines the quantitative change both of the stress state and the deformation state of the material and consequently its elastic-plastic behavior. Using higher tension during the process of plastic bending in wire passing between successive misaligned dies results to a smaller wire-bending radius; hence, the plastic strain in a direction perpendicular to the axis of the wire responsible for the mechanism of shear is larger and leads to a localized plastic flow in the shear bands. On the other hand, low tension causes the CDT process of wire to take place with greater participation of elastic strain (that is, the plastic strain part is smaller), which favors the dominance of homogeneous deformation. These situations are distinctly different from that occurring during the uniaxial tensile test.

It should also be noted that CDT improves the technological characteristics only for wires with sufficient quality, i.e., with no significant internal defects (pores, voids, discontinuities, micro-cracks, lack of sufficient continuity adhesion at grain boundaries, etc.) resulting from the production of wire by powder metallurgy. Otherwise, the increasing scatter of obtained value of twists $\mathrm{N}_{\mathrm{T}}$ along with an increase in the number of passes indicates a deepening differentiation of wire structure in its various parts (at length) thus may also serve as a useful criterion for assessing the quality of a commercial wire. These problems will be the subject of future investigations.

\section{Conclusions}

(1) CDT of tungsten wires improves their technological properties, as defined in the torsion with tension test by $\mathrm{N}_{T}$ before fracturing.

(2) The increase of the $\mathrm{N}_{\mathrm{T}}$ index by about $20 \%$ is reached by a wire subjected to two series of CDT.

(3) The magnitude of the wire tension in the process of CDT has a significant impact on its technological properties defined by the value of the $\mathrm{N}_{\mathrm{T}}$ index. Its highest value was reached by a wire subjected to slight tension (tensile stress).
(4) Using both higher tension and more cycles of multilateral bending with shearing is not justified because it only results in stabilizing the previously reached value of the $\mathrm{N}_{\mathrm{T}}$ index.

(5) The significant improvement of technological properties of the CDT tungsten wire is not accompanied by a change of mechanical parameters determined in tensile test, confirming the held belief in the low usability of such test to anticipate the technological behavior of the wire.

Obtained experimental results demonstrate the suitability CDT of tungsten wire for the production of spirals. In particular, based on the method used for successively advancing the wire length, cyclic, multidirectional bending with little strain, causes relaxation of the internal stresses. For this reason, there is a restriction to the tendency of the wire breaking during the plastic forming of spirals. As a result, it was confirmed that reducing the amount of waste under manufacturing conditions increases the efficiency of the production process by eliminating downtime automated production equipment in which, after breaking the wire, an emergency stop must be "manually" activated by the operator.

Acknowledgments This work was supported by the Polish Ministry of Education and Science and Higher Education under project No UMO-2011/03/B/ST8/05365 (AGH: 18.18.180.610).

Open Access This article is distributed under the terms of the Creative Commons Attribution License which permits any use, distribution, and reproduction in any medium, provided the original author(s) and the source are credited.

\section{References}

1. Bochniak W, Mosor N (1985) The influence of cyclic reversed bending on structure and mechanical properties of B6 tin bronze strips. Metall Foundry 11:455-474

2. Walter JL, Briant CL, Koch EE (1982) Splitting of tungsten wire in the knife-edge compression test. Metall Trans A13:1501-1510

3. Bochniak W, Pieła K, Filipek P (2010) Method of evaluation of wire quality and the device for executing this method. Patent application No PL386513 A1.

4. Bochniak W, Pieła K, (2007) Unpublished work

5. Filipek P (2010) Develop methods and criterion for identifying quality tungsten wires used for elements of the discharge light sources. Ph.D. thesis, AGH University of Science and Technology, Cracow

6. Bochniak W, Pieła K (2011) Method of betterment technological properties of winding wires. Patent application No P-395 196

7. Winter AT (1974) A model for the fatigue of copper at low plastic strain amplitudes. Philos Mag 30:719-738

8. Huang JY, Zhu YT, Jiang H, Lowe TC (2001) Microstructures and dislocation configurations in nanostructured $\mathrm{Cu}$ processed by repetitive corrugation and straightening. Acta Materlialia 49:1497-1505

9. Emmens WC, van Boogaard AH (2011) Cyclic stretch-bending. J Mater Process Technol 211:1965-1981

10. Valiev RZ, Krasilnikov NA, Tsenev NK (1991) Plastic deformation of alloys with submicron-grained structure. Mater Sci Eng A137:35-40

11. Bochniak W, (2012) unpublished results 